\title{
Dual self-image technique for beam collimation
}

\author{
Jose Maria Herrera-Fernandez ${ }^{1}$, Luis Miguel Sanchez-Brea ${ }^{1}$, \\ Francisco Jose Torcal-Milla ${ }^{1}$, Tomas Morlanes ${ }^{2}$ and Eusebio \\ Bernabeu $^{1}$ \\ ${ }^{1}$ Optics Department, Applied Optics Complutense Group, Universidad Complutense \\ de Madrid (UCM), Facultad de Ciencias Físicas, Plaza de las Ciencias 1, 28040, \\ Madrid, Spain. \\ ${ }^{2}$ AOTEK S. Coop., B ${ }^{\circ}$ San Andrés 19, 20500, Mondragón, Guipuzcoa, Spain. \\ E-mail: jmherrer@ucm.es
}

\begin{abstract}
We propose an accurate technique for obtaining highly collimated beams, which also allows testing the collimation degree of a beam. It is based on comparing the period of two different self-images produced by a single diffraction grating. In this way, variations in the period of the diffraction grating do not affect to the measuring procedure. Self-images are acquired by two CMOS cameras and their periods are determined by fitting the variogram function of the self-images to a cosine function with polynomial envelopes. This way, loss of accuracy caused by imperfections of the measured self-images is avoided. As usual, collimation is obtained by displacing the collimation element with respect to the source along the optical axis. When the period of both self-images coincides, collimation is achieved. With this method neither a strict control of the period of the diffraction grating nor a transverse displacement, required in other techniques, are necessary. As an example, a LED considering paraxial approximation and point source illumination is collimated resulting a resolution in the divergence of the beam of $\delta \phi= \pm 1.57 \mu \mathrm{rad}$.
\end{abstract}

Keywords: Talbot effect, self-imaging, collimation, diffraction

\section{Introduction}

Highly collimated beams are necessary for many optical experiments and applications, such as metrology, information processing, instrumentation, lithography, holography, etc. A simple technique for a rough estimation is auto-collimation, where the collimation is achieved by comparing the size of the source with its image. Nevertheless, results are quite imprecise since the intensity distribution of the light beam usually changes as it propagates. More accurate beam collimation techniques based on different optical effects have been proposed such as those based on interferometry $[1,2,3,4,5,6,7,8,9]$. Selfimaging-based methods, also known as Talbot interferometry, use Talbot effect as a tool to create moiré fringes at the output of a double grating system where the second grating is placed at a self-image plane of the first one $[10,11,12], z_{T}=2 l p^{2} / \lambda$, where $l$ is an integer, $p$ is the period of the grating, and $\lambda$ is the wavelength [13]. This 




Figure 1. Scheme of the collimation method based on grating self-imaging. When the beam is not collimated, the period of the self-images changes with the distance. However, when the beam is collimated, the period of the self-images is equal to that of the grating. $\Delta z$ is the distance between the source $s$ and the focal point of the lens whose focal length is $f$ and whose principal plane is $H, z_{0}$ is the distance between $s$ and $H, z_{1}$ is the distance from $H$ to the diffraction grating $G$ whose period is $p$, $z_{2}=l z_{T}$ is the distance from $G$ to a CMOS sensor placed at one self-image plane and finally, $p_{z}$ is the period of the signal acquired by CMOS sensor.

setup has been modified in different ways, by changing the periods of the gratings, by transverse shifting one of the gratings, etc $[14,15,16]$. Another method consists of replacing one of the linear gratings by a circular grating [16]. Unlike the former case, the collimation degree is obtained with a complex analysis of the parabolic fringes formed at the observation plane. The main advantage is that a continuous transverse displacement of the grating is not required. On the other hand, a simple technique to check the collimation degree of a beam through the Lissajous figure produced by a dual diffractive optical system is shown in [17]. In this case, the second element is formed by a mask with two diffraction gratings with the same period $p$, laterally shifted $p / 4$, and located at a Talbot distance of the first grating. Following, in order to avoid a continuous movement of the grating, a complex mask is proposed in [18] so that several points of the Lissajous figure are obtained simultaneously. In both cases the collimation degree is determined by measuring the ellipticity of the Lissajous figure.

A very simple technique, where only one diffraction grating is required to measure the collimation degree of the beam, has been recently developed [19]. It takes advantage of the low cost and small size of photodetectors in CCD and CMOS cameras. A scheme of the set-up is shown in figure 1.

When the beam passes through the diffraction grating $G$ whose period is $p$, selfimages with maximum contrast are located at multiples of the Talbot distance $z_{T}$. The beam is collimated when the period of the grating and the period of the self-image at the sensor, $p_{z}$, are equal. This happens when the distance from the light source $s$ to the focal point of the lens is null, $\Delta z=\left|z_{0}-f\right|=0$, where $z_{0}$ is the distance from the lens to $s$ and $f$ is the focal length of the collimating lens. Otherwise, the beam is not 
collimated and $\Delta z$ is given by

$$
\Delta z=-\frac{f^{2}}{z_{2} p} \Delta p
$$

being $\Delta p=p_{z}-p$ the variation of $p_{z}$ with respect to the period of the grating. As a consequence, a precise estimation of the period of the grating and the self-image is required to determine the collimation point. For this task the variogram function is used.

The period of the diffraction grating can be known with high accuracy. Nevertheless, a tiny change of the temperature, which induces a dilation of the grating, sensor, opto-mechanical components, etc, or slight rotations of the grating or the CMOS camera, may change the period of the grating and the self-images. These small variations in the period of the grating are very prejudicial for determining the collimation of the light beam. Therefore, a more robust procedure is required.

In this work, we propose a self-imaging technique with a single diffraction grating where neither transverse displacement of the grating nor a strict control of its period are required. The collimated beam is achieved by measuring the period of two different self-images with two CMOS cameras or two linear arrays of photodetectors. We use the variogram function to determine the period of the self-images with high accuracy. We take advantage of the property that imperfections on the intensity distribution appears in the variogram as a polynomial variation of the envelopes. When both selfimages present the same period, the beam results collimated. As we demonstrate in the following sections, the results show high accuracy and the collimation position can be found easily and rapidly.

\section{Beam collimation technique}

Let us consider the two cameras scheme depicted in figure 2. A point and monochromatic incident light source $s$, whose mean wavelength is $\lambda$, illuminates a system formed by a lens whose focal length is $f$, an amplitude diffraction grating whose nominal period is $p$, a beam splitter $B S$ and a couple of CMOS cameras, $C_{z 2}$ and $C_{z 3}$. The distance from the source $s$ to the lens $L$ is $z_{0}$, from $L$ to $G$ is $z_{1}$, and the distances from $G$ to $C_{z 2}$ and $C_{z 3}$ are $z_{2}$ and $z_{3}$, respectively. Thus, a system with two arms for the simultaneous measurement of the self-images of the same area of $G$ is configured.

The intensity distributions at $z_{2}$ and $z_{3}$ distances are obtained using Fresnel approach, [17],

$$
\begin{aligned}
& I\left(x_{3}, z_{2}\right) \propto I_{0} \sum_{n, n^{\prime}} a_{n} a_{n^{\prime}}^{*} \exp \left[i \frac{q}{1+\alpha z_{2}}\left(n-n^{\prime}\right) x_{3}\right] \exp \left[-i \frac{q^{2}}{2 k}\left(n^{2}-n^{\prime 2}\right) \frac{z_{2}}{1+\alpha z_{2}}\right. \\
& I\left(x_{3}, z_{3}\right) \propto I_{0} \sum_{n, n^{\prime}} a_{n} a_{n^{\prime}}^{*} \exp \left[i \frac{q}{1+\alpha z_{3}}\left(n-n^{\prime}\right) x_{3}\right] \exp \left[-i \frac{q^{2}}{2 k}\left(n^{2}-n^{\prime 2}\right) \frac{z_{3}}{1+\alpha z_{3}}\right],
\end{aligned}
$$

where $a_{n}$ are the Fourier coefficients of the grating, * indicates complex conjugate, $q=2 \pi / p, k=2 \pi / \lambda, n$ and $n^{\prime}$ are integers, $i=\sqrt{-1}$ is the imaginary unit, $I_{0}$ is the intensity distribution of the input beam, $z_{2}$ and $z_{3}$ represent distances from the grating to the hypothetical observation planes, $x_{3}$ is the coordinate parallel to $G$, and 


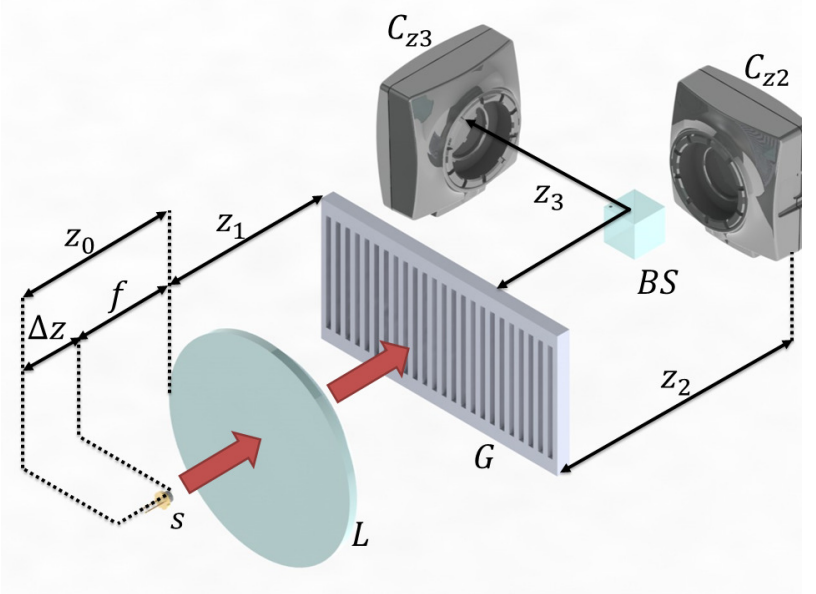

Figure 2. Experimental setup for beam collimation. $s$ is the light source which is moved with a stepper motor along the optical axis, $L$ is a converging lens whose focal length is $f, G$ is an amplitude Ronchi diffraction grating whose nominal period is $p$, $B S$ is a beam splitter, $C_{z 2}$ y $C_{z 3}$ are two CMOS cameras, $z_{0}$ is the distance between $s$ and $L, \Delta z=\left|z_{0}-f\right|, z_{1}$ is the distance from $L$ to $G$, and $z_{2}$ and $z_{3}$ are the distances from $G$ to $C_{z 2}$ and $C_{z 3}$ respectively.

$\alpha=1 / z_{1}-1 /\left(z_{1}-z_{1}^{2} \Delta z / f^{2}\right) \approx-\Delta z / f^{2}$ is the collimation degree. The collimation degree depends linearly on the distance $\Delta z$ from the light source to the focal point of the lens. According to equation (2), the collimation degree of the light beam affects both to the period and location of the self-images [17]. Considering the first exponential terms in equation (2), the periods of the fringes at $C_{z 2}$ and $C_{z 3}$ are given by

$$
\begin{aligned}
& p_{z 2}=\left(1+\alpha z_{2}\right) p, \\
& p_{z 3}=\left(1+\alpha z_{3}\right) p,
\end{aligned}
$$

respectively. Then, even when the period of the grating $p$ is unknown, we can obtain the collimation degree through the quotient between both expressions,

$$
\alpha=-\frac{p_{z 2}-p_{z 3}}{p_{z 2} z_{3}-p_{z 3} z_{2}} .
$$

Considering the definition of the collimation degree, $\alpha \approx-\Delta z / f^{2}$ of the light source, we obtain

$$
\Delta z=-\alpha f^{2}=\frac{p_{z 2}-p_{z 3}}{p_{z 2} z_{3}-p_{z 3} z_{2}} f^{2} .
$$

It is important to notice that the CMOS cameras can be placed within an interval around the Talbot planes as long as the visibility of the fringes is high enough to determine the period [20]. This is not usually a problem since self-images remain quasi constant along a range around every Talbot plane [18]. To obtain collimation, $\Delta z=0$, we need that the period of the fringes at both observations planes is the same. According to equation (5), when the distances $z_{2}$ and $z_{3}$ are different, this can be fulfilled only if $p_{z 2}=p_{z 3}=p$. 


\section{Experimental results}

The key to find the collimation point with accuracy is to measure

self-images simultaneously until both match. In the experimental setup depicted in figure 2 we have used an IR LED model AP2012SF4C whose wavelength is $\lambda=880 \mathrm{~nm}$, a converging lens whose diameter is $D=17 \mathrm{~mm}$ and focal length is $f=40 \mathrm{~mm}$, a diffraction grating $G$ whose nominal period is $p=100 \mu \mathrm{m}$ with a manufacturing error of $3 \mu \mathrm{m} / \mathrm{m}$, and two Imaging Source cameras model DMK72BUC02 whose pixel size is $2.2 \mu \mathrm{m} \times 2.2 \mu \mathrm{m}$ whose chips have been considered equal. Also, a high-precision stepper motor whose minimum incremental motion is $0.3 \mu \mathrm{m}$ is used to move the light source along the optical axis and place it at the focal plane of the lens. We have chosen as distances to the self-images (distances from the grating to the cameras) $z_{2}=2 p^{2} / \lambda=22.72 \mathrm{~mm}$ and $z_{3}=5 p^{2} / \lambda=56.81 \mathrm{~mm}$ but other distances would be also valid provided that $z_{2} \neq z_{3}$. Notice that we have used one even self-image and one odd self-image. This fact does not influence the result since, despite the contrast is inverse between them, the period behavior is the same. Furthermore, the perpendicularity to the optical axis and the alignment of the slits of the self-images respect to the rows or columns of the sensors have been checked. An example of the intensity distribution and fringes profile acquired by both CMOS cameras is shown in figure 3 . In this case the beam is not collimated. On the other hand, to minimize errors due to nonperpendicularity between cameras, we have used cube beam splitters and reflections of the beam to align them with high accuracy.

The intensity distribution of the self-images usually presents imperfections which are harmful for determining the period as we can see in figures 3(c) and (d) where the integration over columns of the intensity is not perfectly sinusoidal. As a consequence, we have chosen the variogram function in order to accurately determine the period of the self-images [19]. It has been proven as a very useful technique for improving the quality of fringes and eliminating residual noise in optical imaging and other areas [21, 20]. The variogram function is defined as [22]

$$
2 \gamma(h)=\left\langle[I(x+h)-I(x)]^{2}\right\rangle_{x},
$$

where \langle\rangle$_{x}$ is the spatial average with respect to $x, I(x)$ is the intensity at $x$, and $h$ is the distance between two measured points. In the case of a regularly sampled signal, such as the signal obtained with a linear array of photodiodes, equation (6) becomes [20]

$$
2 \gamma(h=n \Delta x)=\frac{1}{N-n} \sum_{i=1}^{N-n}\left(I_{i+n}-I_{i}\right)^{2},
$$

where $N$ is the total number of pixels, $I_{i}=I(i \Delta x)$ is the intensity detected in the pixel $i$ and $\Delta x$ is the distance between two adjacent pixels of the discrete variogram.

The variogram of a periodic signal is also periodic with the same period than the original signal. In addition, due to the averaging process performed in equation (6), the variogram function is much smoother than the original intensity distribution. Influence of grating defects on the visibility of the fringes has been reported by the authors in 
(a)

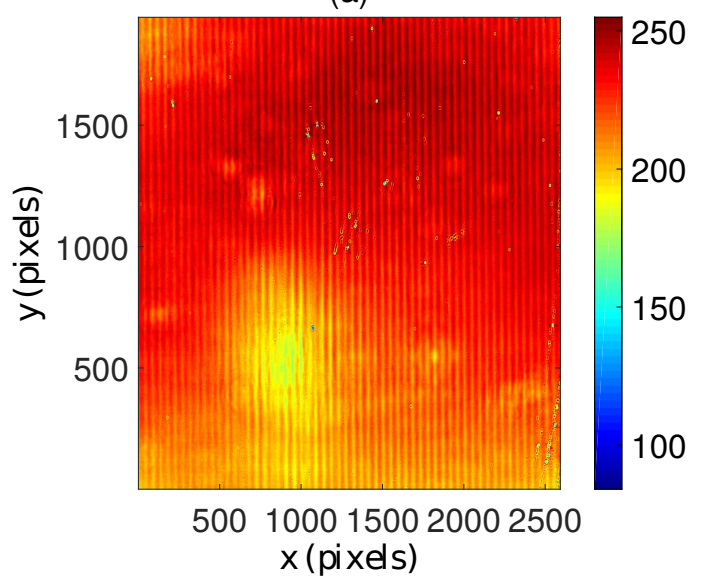

(c)

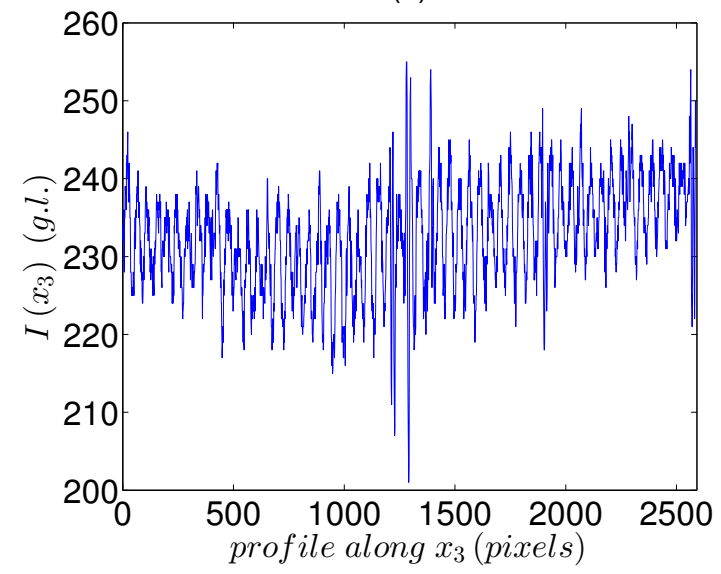

(b)



(d)

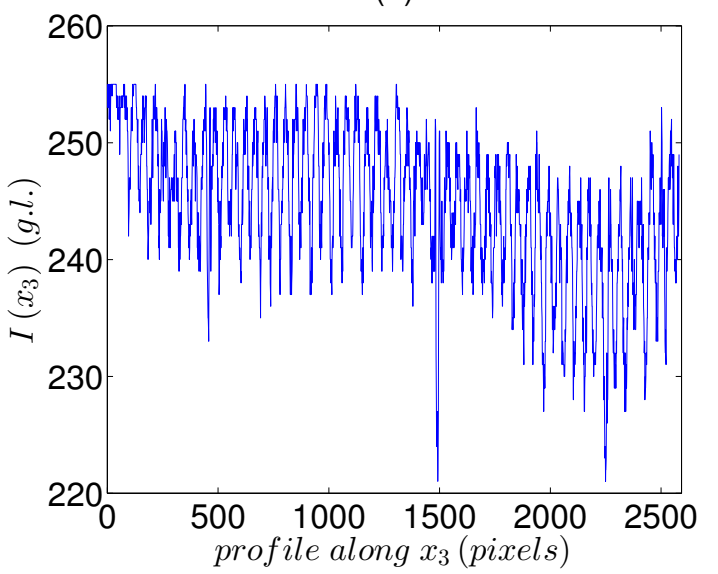

Figure 3. (a) and (b) Intensity distribution obtained with both CMOS cameras and (c) and (d) examples of an intensity profile obtained as a vertical integration of the intensity carpets, at an arbitrary position of the light source when $z_{2}=22.72 \mathrm{~mm}$ and $z_{3}=56.81 \mathrm{~mm}$, respectively. Both intensity profiles present imperfections that can cause a loss of accuracy in the measurement of the collimation distance. $x$ and $y$ represent the pixels of the CMOS sensors being the pixel size $2.2 \mu \mathrm{m} \times 2.2 \mu \mathrm{m}$.

previous works $[23,24]$. An example is shown in figure 4 where the variogram function has been obtained for two sections of figure 3. Although the profile intensity distribution at the self-images is quite fluctuating, figures $4(\mathrm{a})$ and $4(\mathrm{~b})$, the variogram function is very smooth, solid line in Figs. 4(c) and 4(d).

Noise and random fluctuations in the experimental signal are translated into the variogram as a variation of its envelopes. A possible way to obtain the period of the variogram is by determining the location of the minima using a polynomial fitting [19]. Nevertheless, it is possible to obtain better results when the complete variogram function is used. We have checked several solutions and we have found that the best option to fit the variogram in this particular case is by using a periodic function where the envelopes are third power polynomials

$$
2 \gamma(h)=\left(a_{1}+b_{1} h+c_{1} h^{2}+d_{1} h^{3}\right)-\left(a_{2}+b_{2} h+c_{2} h^{2}+d_{2} h^{3}\right) \cos \left(\frac{2 \pi}{p} h\right),
$$


(a)



(c)



(e)

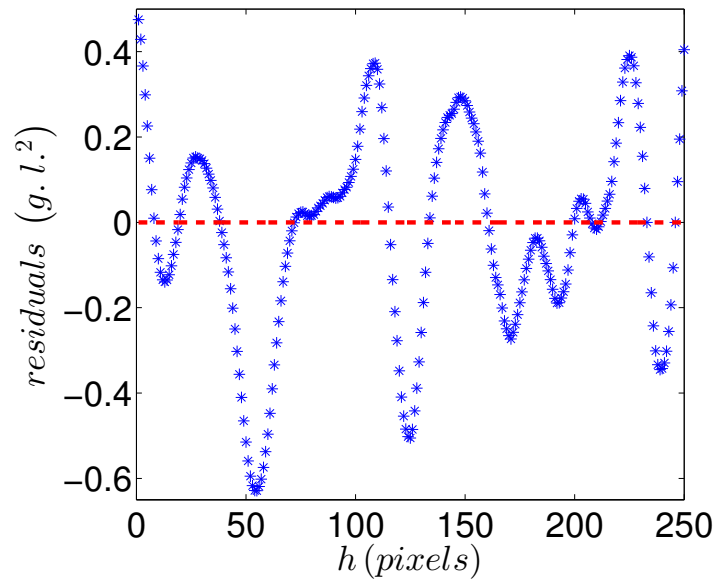

(b)

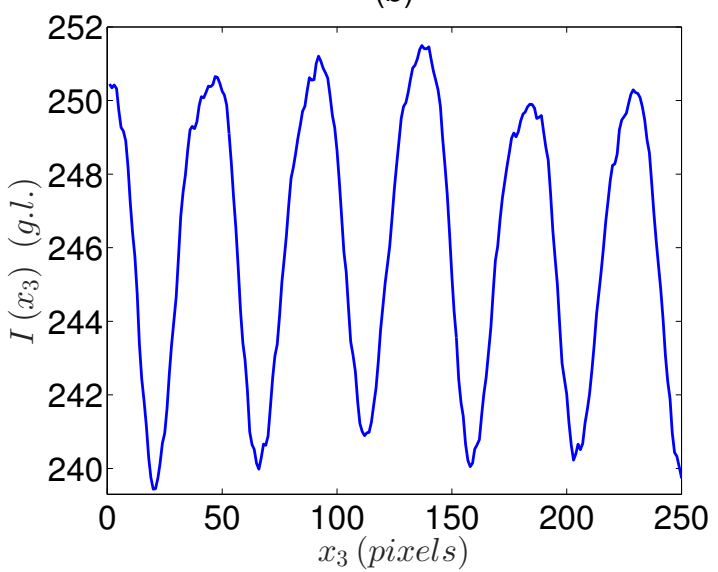

(d)

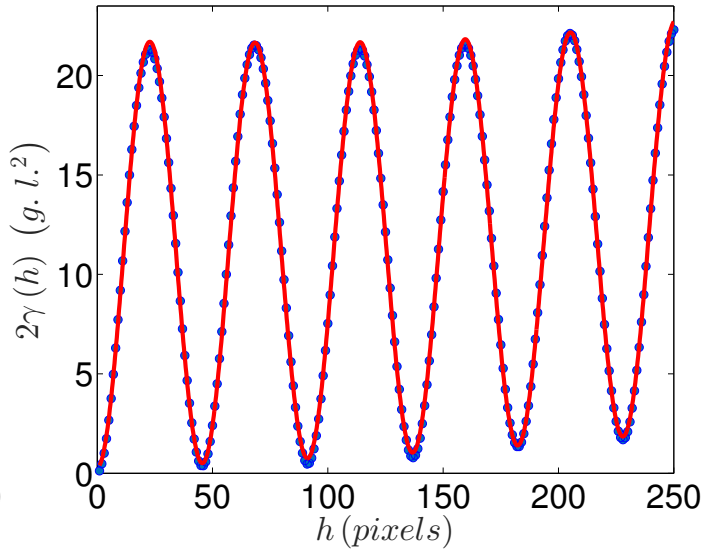

(f)

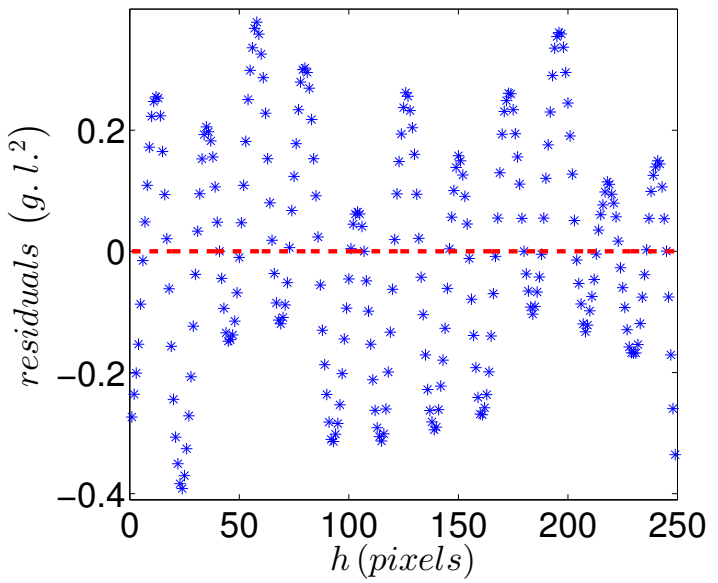

Figure 4. (a) and (b) two sections of Figs. 3(c) and 3(d), (c) and (d) variogram functions (red solid line) obtained from (a) and (b), respectively and their fittings to equation (8) (blue $\bullet$ ). (e) and (f) are the residuals of the fitting for both cases. 
where $a_{k}, b_{k}, c_{k}, d_{k},(k=1,2)$, and $p$ are free parameters. The fittings are shown in Figs. 4(c) and 4(d) (blue $\bullet$ ). The R-square parameters are $R^{2}=0.9995$ for the first case and $R^{2}=0.9994$ for the second case, values that are kept or increased throughout the experiment. In Figs. 4(e) and 4(f) we can also see the residuals of the experimental variograms to equation (8). The root mean squared error (RMSE) is $0.24 \mathrm{g.l}^{2}$ and 0.19 g.l. ${ }^{2}$ respectively. For this example, we have obtained that the periods of both self-images result $p_{z 2}=100.39 \mu \mathrm{m}$ and $p_{z 3}=100.31 \mu \mathrm{m}$. Using equation (4), we obtain that the collimation degree is $\alpha=-2.26 \cdot 10^{-8} \mu \mathrm{m}^{-1}$ and the distance between the light source to the focal plane for this particular case is $\Delta z=36.12 \mu \mathrm{m}$.

In order to obtain a highly collimated beam, the earlier process has been repeated for different positions of the light source. For this, we have displaced $s$ with respect to the collimating lens using the linear stepper motor along $200 \mu \mathrm{m}$. We have determined the period of the self-images for each location (100 measurements into the interval $z=27.68-27.88 \mathrm{~mm}$ where these values are arbitrary absolute positions of the linear stepper motor along z-axis). In figure 5(a), the variation of the experimental periods, $p_{z}$, of each image acquired by both cameras is shown. Since the distances of the two CMOS cameras to the grating are different, the slopes of the $p(z)$ functions are different and both figures coincide for a certain distance $z_{c}$ that corresponds to the unknown collimation position, $\Delta z=0$.

(a)



(b)

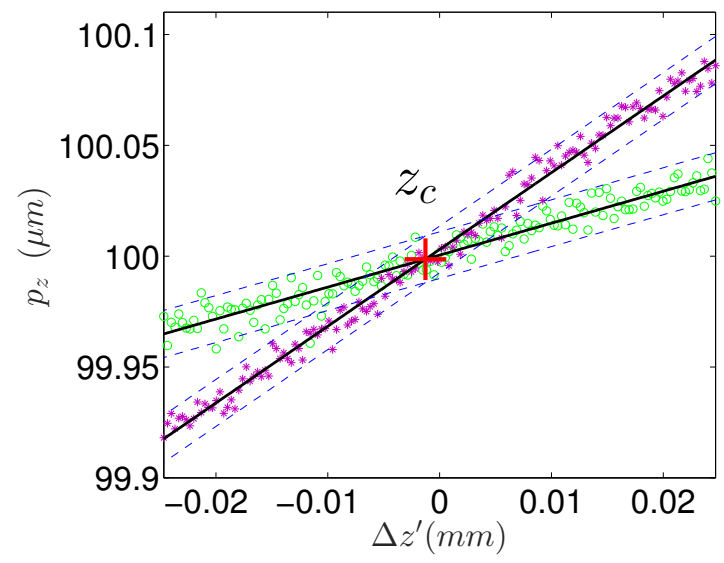

Figure 5. Variation of the experimental period of each self-image with the distance in both cameras. Green $\circ$ are the experimental period measured by $C_{z 2}$, purple * are the experimental period measured by $C_{z 3}$. (a) 100 measurements into the interval $z=27.68-27.88 \mathrm{~mm}$ where $z$ values are arbitrary absolute positions of the linear stepper motor along z-axis, (b) 150 measurements around the approximate point of intersection of $(\mathrm{a}), z_{c} \approx 27.784 \mathrm{~mm}$, that is, $\Delta z^{\prime}=z_{c} \pm 25 \mu \mathrm{m}$ and $p_{z}$ is the period of the self-images. The collimation position $($ red +$)$ is obtained through the intersection of the linear fittings of both experimental data sets (black solid lines) being $z_{c}=-1.3 \pm 0.3 \mu \mathrm{m}$. Blue dashed lines represent the $95 \%$ confidence intervals.

In figure $5(\mathrm{a})$ this position is $z_{c} \approx 27.784 \mu \mathrm{m}$. Once the approximated point of intersection is known, a new set of 150 experimental measurements is obtained around 


\begin{tabular}{|c|c|c|c|c|}
\hline Camera & $m(\mu m / m m)$ & $n(\mu m)$ & $R^{2}$ & $R M S E(n m)$ \\
\hline$C_{z 2}$ & $1.44 \pm 0.06$ & $100.0005 \pm 0.0008$ & 0.9388 & 5.3 \\
\hline$C_{z 3}$ & $3.46 \pm 0.05$ & $100.0030 \pm 0.0007$ & 0.9904 & 4.9 \\
\hline
\end{tabular}

Table 1. Goodness of the fittings shown in figure 5(b) for the measurements of both sensors. $m$ is the slope, $n$ is the intercept, $R^{2}$ is the square of the correlation between the response values and the predicted response values, and $R M S E$ is the root mean square of the error.

$z_{c}$, that is, $\Delta z^{\prime}=z_{c} \pm 25 \mu \mathrm{m}$, figure $5(\mathrm{~b})$. Note that since both lines have slopes different, a final device could carry out the beam collimation with a few points around $z_{c}$. This reduces execution time to a few seconds with a current computer.

Since an increment of accuracy is desired, a least squares fitting of the experimental values, $\circ$ and $*$ in figure $5(\mathrm{~b})$, to a linear equation is performed [25], black solid lines in figure $5(\mathrm{~b})$,

$$
p_{i}^{\prime}=m_{i}(z-\langle z\rangle)+n_{i},
$$

being $m$ and $n$ free parameters, $\langle z\rangle$ the mean of $z$, and $i=1,2$. Also, the $95 \%$ confidence intervals for goodness of each fitting (blue dashed lines) are shown. The results of the linear fittings are shown in table 1 . In both cases, the quality parameters indicate good fittings, being the R-square close to 1 and the root-mean-square deviations relatively small.

Finally, the intersection of both fittings has been estimated and, thus, the resolution in the collimation distance of the IR-LED has been obtained. The intersection point is given by solving the system of equations consisting of $p_{1}^{\prime}=p_{2}^{\prime}$,

$$
m_{1}(z-\langle z\rangle)+n_{1}=m_{2}(z-\langle z\rangle)+n_{2} .
$$

For this calculation, we have used the Python module Pycse which has the advantage of providing the error directly through the propagation of uncertainties [26]. The collimation point is located at $z_{c}=-1.3 \pm 0.3 \mu \mathrm{m}$. Following, we may calculate the minimum beam divergence that we can obtain for a point source. Since the lens equation is given by

$$
\frac{1}{s^{\prime}}-\frac{1}{s}=\frac{1}{f}
$$

where $s=-f+\delta z_{c}$ and $\delta z_{c}$ is the uncertainty in the location of the source, the divergence of the IR-LED is given by

$$
\delta \phi=\frac{D}{2 s^{\prime}} \approx \frac{D \delta z_{c}}{2 f^{2}},
$$

being $D=17 \mathrm{~mm}$ and $f=40 \mathrm{~mm}$ the diameter and the focal distance of the lens, respectively. Thus, the resolution in the divergence of the beam results $\delta \phi= \pm 1.57 \mu \mathrm{rad}$. Classical methods in which the interference is generated by, for example, a planeparallel plate, are fairly simple and precise. More recently, Chang in [?] proposes a collimation testing and calibration using a heterodyne Moiré method. This method 
is fast, relatively simple and achieves a positioning accuracy close to $\pm 7 \mu \mathrm{m}$. On the other hand, the technique proposed by Disawal is based on Lau effect and achieves collimation of incoherent beams with an accuracy of $\pm 1 \mu m$ [?]. Talbot interferometry is used suitably modified by Patorsky to prevent the continued displacement of the second grating. In this case, the accuracy is $\pm 20 \mu \mathrm{m}$ [16]. Also based on the Talbot effect, Wang obtained accuracy of $\pm 10^{-7} \mathrm{rad}$ when he collimates monochromatic beams with a lens of focal distance $f=50 \mathrm{~mm}$ [?]. With respect to our previous work, The resolution in the divergence of the beam obtained with the proposed method represents a remarkable result compared to [19, 18] where the divergence of the beam is $10 \mu \mathrm{rad}$ and $4.16 \mu \mathrm{rad}$, respectively.

As a remark, when the light source presents a finite size, $l$, then the divergence of the beam is increased by the term $\delta \phi=l / 2 f^{\prime}$, which cannot be reduced with this technique. In this case, our technique produce the optimum divergence for extent light sources.

Note that to reach the collimation distance a high number of measurements as in

figure 5 is not required. To reduce the execution time to a few seconds we can perform the following procedure:

(i) In the first place, we determine the period of the two self-images acquired with cameras for an unknown location $\Delta z_{(1)}$, and following we estimate the value $\Delta z$ for this location using equation (5).

(ii) Then, we move to this location and we determine again the periods $p_{z 2}, p_{z 3}$ and $\Delta z_{(2)}$.

(iii) We repeat step (ii) until the periods $p_{z 2}, p_{z 3}$ are close enough with respect the uncertainty in the location.

\section{Conclusions}

In this work, we present a dual self-image based collimation technique which consists of the comparison of periods of two different self-images produced by a single diffraction grating and acquired by two CMOS cameras at two different Talbot distances. Then, variations in the period of the diffraction grating produced by rotations, temperature variation, etc, do not affect to the measuring technique. We have used the variogram function in order to reduce the uncertainty in the estimation of the experimental periods due to random fluctuations. Afterwards, the accuracy in the experimental measure of the periods is increased by fitting the complete variogram to a certain experimental function. Finally, each set of experimental periods is fitted to a straight line. As a consequence the collimation point is obtained by determining the intersection of both fittings. Unlike other methods, neither a strict knowledge of the period of the diffraction grating nor a transverse displacement of the grating are needed. As an example of application of the technique, an IR LED is used as light source and two CMOS cameras are used to acquire the self-images produced by the diffraction grating of period $p=100 \mu \mathrm{m}$ at 
$z_{2}=2 p^{2} / \lambda=22.72 \mathrm{~mm}$ and $z_{3}=5 p^{2} / \lambda=56.81 \mathrm{~mm}$. As collimation element, a lens whose diameter is $D=17 \mathrm{~mm}$ and whose focal length is $f=40 \mathrm{~mm}$ has been used. With this experimental setup we have achieved a resolution in the divergence of the beam of $\delta \phi= \pm 1.57 \mu \mathrm{rad}$.

\section{Acknowledgments}

The authors thank to O. J. Casas for his valuable comments. This work has been supported by project DPI2011-27851 of the Ministerio de Economía y Competitividad of Spain and the SEGVAUTO-TRIES Tecnologías 2013 CM S2013/MIT-2713 program of the Comunidad de Madrid.

\section{References}

[1] M.V.R.K. Murty. The use of a single plane parallel plate as a lateral shearing interferometer with a visible gas laser source. Appl. Opt., 3(4):531-534, 1964.

[2] D. Joyeux and Y. Cohen-Sabban. High magnification self-imaging. Appl. Opt., 21(4):625-627, 1982.

[3] M.P. Kothiyal and R.S. Sirohi. Improved collimation testing using talbot interferometry. Appl. Opt., 26(19):4056-4057, 1987.

[4] K. Patorski. I the self-imaging phenomenon and its applications. Prog. Optics, 27:1-108, 1989.

[5] A.R. Ganesan and P. Venkateswarlu. Laser beam collimation using talbot interferometry. Appl. Opt., 32(16):2918-2920, 1993.

[6] J. Choi, G.M. Perera, M.D. Aggarwal, R.P. Shukla, and M.V. Mantravadi. Wedge-plate shearing interferometers for collimation testing: use of a moiré technique. Appl. Opt., 34(19):3628-3638, 1995.

[7] J.S. Darlin, M.P. Kothiyal, and R.S. Sirohi. A phase-conjugate twyman-green interferometer with increased sensitivity for laser beam collimation. J. Mod. Opt., 45(11):2371-2378, 1998.

[8] D. Malacara. Optical Shop Testing. Wiley Series in Pure and Applied Optics. Wiley, 2007.

[9] S. Prakash, S. Rana, S. Prakash, and O. Sasaki. Automated collimation testing using a temporal phase shifting technique in talbot interferometry. Appl. Opt., 47(31):5938-5943, 2008.

[10] D.E. Silva. A simple interferometric method of beam collimation. Appl. Opt., 10(8):1980_1-1982, 1971.

[11] S. Yokozeki, K. Patorski, and K. Ohnishi. Collimation method using fourier imaging and moiré techniques. Opt. Commun., 14(4):401-405, 1975.

[12] K.V. Sriram, M.P. Kothiyal, and R.S. Sirohi. Self-referencing collimation testing techniques. Optical Engineering, 32(1):94-100, 1993.

[13] H.F. Talbot. Lxxvi. facts relating to optical science. no. iv. The London and Edinburgh Philosophical Magazine and Journal of Science, 9(56):401-407, 1836.

[14] C. Shakher, S. Prakash, D. Nand, and R. Kumar. Collimation testing with circular gratings. Appl. Opt., 40(8):1175-1179, 2001.

[15] L. Huang and X. Su. Method for acquiring the characteristic parameter of the dual-spiral moiré fringes. Opt. Lett., 33(8):872-874, 2008.

[16] K. Patorski, K. Pokorski, and M. Trusiak. Circular-linear grating talbot interferometry with moiré fresnel imaging for beam collimation. Opt. Lett., 39(2):291-294, 2014.

[17] L.M. Sanchez-Brea, F.J. Torcal-Milla, F.J. Salgado-Remacha, T. Morlanes, I. Jimenez-Castillo, and E. Bernabeu. Collimation method using a double grating system. Appl. Opt., 49(17):3363$3368,2010$. 
[18] F.J. Torcal-Milla, L.M. Sanchez-Brea, and J.M. Herrera-Fernandez. Lissajous figure-based singleframe collimation technique. Sensors and Actuators A: Physical, 233:259 - 266, 2015.

[19] L.M. Sanchez-Brea, F.J. Torcal-Milla, J.M. Herrera-Fernandez, T. Morlanes, and E. Bernabeu. Self-imaging technique for beam collimation. Opt. Lett., 39(19):5764-5767, 2014.

[20] L.M. Sanchez-Brea, F.J. Torcal-Milla, and E. Bernabeu. Variogram-based method for contrast measurement. Appl. Opt., 46(22):5027-5033, Aug 2007.

[21] L.M. Sanchez-Brea and E. Bernabeu. Estimation of the standard deviation in three-dimensional microscopy by spatial statistics. J. Microscopy - Oxford, 218:193-197, 2005.

[22] N.A.C. Cressie. Statistics for Spatial Data, revised edition, volume 928. Wiley, New York, 1993.

[23] L.M. Sanchez-Brea and F. J. Torcal-Milla. Near-field diffraction of gratings with surface defects. Applied Optics, 49(11):2190-2197, 2010.

[24] F.J. Torcal-Milla, I. Harder, and N. Lindlein. Effect of fabrication errors on the diffraction pattern produced by sawtooth gratings. Applied optics, 49(9):1599-1606, 2010.

[25] IEC BIPM, ILAC IFCC, IUPAP IUPAC, and OIML ISO. Evaluation of measurement data-guide for the expression of uncertainty in measurement. jcgm 100: 2008, 2008.

[26] John Kitchin. pycse: First release, 2015. 